Revue scientifique sur la conception et l'aménagement de l'espace

\title{
Évaluation de la qualité paysagère dans
}

l'aménagement urbain

Le cas de Paris

Landscape quality's and evaluation in urban projets - The case of Paris.

\section{Cedissia About-de Chastenet}

\section{(2) OpenEdition}

Journals

Édition électronique

URL : http://journals.openedition.org/paysage/15509

DOI : 10.4000/paysage. 15509

ISSN : 1969-6124

Éditeur :

École nationale supérieure du paysage de Versailles-Marseille, Institut national des sciences appliquées Centre Val de Loire - École de la nature et du paysage, École nationale supérieure d'architecture et de paysage de Bordeaux, École nationale supérieure d'architecture et de paysage de Lille, Agrocampus Angers

\section{Référence électronique}

Cedissia About-de Chastenet, «Évaluation de la qualité paysagère dans l'aménagement urbain », Projets de paysage [En ligne], 8| 2012, mis en ligne le 01 octobre 2012, consulté le 11 mars 2021. URL : http://journals.openedition.org/paysage/15509; DOI : https://doi.org/10.4000/paysage.15509

Ce document a été généré automatiquement le 11 mars 2021.

Projets de paysage 


\title{
Évaluation de la qualité paysagère dans l'aménagement urbain
}

\author{
Le cas de Paris
}

Landscape quality's and evaluation in urban projets - The case of Paris.

\author{
Cedissia About-de Chastenet
}

1 Partant du principe, généralement admis, que la société parisienne, comme les sociétés européennes, relève des sociétés dites « à paysage » (Berque, 1996) et que, du fait de cet héritage, les questions urbaines et les politiques environnementales contemporaines créent une transition vers d'autres formes de paysages urbains dits durables, cet article propose d'expérimenter de nouveaux modes de suivi et d'évaluation de ces évolutions paysagères en milieu urbain.

2 Plusieurs démarches abordent la question paysagère sous des angles variés. Dans le cadre de cette étude, nous avons fait le choix de partir de l'approche opérationnelle, illustrée par la démarche de la ville de Paris dans son référentiel «Un aménagement durable pour Paris ", et de la croiser avec l'approche institutionnelle, représentée par la Convention européenne du paysage de Florence (2000), et l'approche «culturaliste ", développée notamment par des auteurs comme Alain Roger (1997) ou Augustin Berque (1996).

3 La finalité de notre réflexion étant de pouvoir offrir aux maîtrises d'ouvrages de nouveaux outils de suivi et d'évaluation de la qualité paysagère dans les projets urbains durables.

4 Nous nous proposons d'étudier dans la première partie les modalités de construction des indicateurs de qualité paysagère, puis dans la deuxième partie l'analyse comparative de ces indicateurs dans trois opérations d'aménagement parisiennes. Enfin, dans la troisième et dernière partie, nous montrerons comment cette analyse, par thématique, s'inscrit également dans une approche globale détaillant la prise en compte des objectifs de développement durable dans les projets urbains. 


\section{Construction des indicateurs de qualité paysagère}

5 Nous partageons le constat, évoqué dans la Convention européenne du paysage, suivant lequel « les évolutions [...] des pratiques en matière d'aménagement du territoire [...] et, plus généralement, les changements économiques mondiaux continuent, dans beaucoup de cas, à accélérer la transformation des paysages ».

6 Afin de répondre au souhait des élus locaux et des usagers de «jouir de paysages de qualité et de [pouvoir] jouer un rôle actif dans leur transformation ", pour préserver le "bien-être individuel et social ", plusieurs outils ont été mis en place par les différents pays s'intéressant notamment aux « évaluations des effets paysagers des interventions [...]». Ces outils s'appuient sur quatre pistes d'action :

- «montrer la manière dont le projet est introduit dans les différents contextes [...]; le raccord avec les matériaux, les couleurs, les techniques de construction [...] ;

- présenter l'état des lieux [...]; démontrer la cohérence entre les caractéristiques du projet et les contextes; simuler l'état des lieux après l'intervention;

- démontrer la conformité du projet par rapport aux objectifs de qualité paysagère des instruments de planification et d'aménagement paysager (plans, chartes, etc.) ;

- évaluer les effets des transformations proposées sur les lieux et introduire, si nécessaire, des mesures d'atténuation qui garantissent le maintien d'une bonne qualité paysagère des lieux et des mesures de compensation qui contribuent à la qualité environnementale. »

7 Nous traduirons ces pistes par quatre objectifs opérationnels : la cohérence du projet dans son contexte urbain, le diagnostic initial, la prise en compte des contraintes politiques et réglementaires propres au territoire dans lequel s'inscrit le projet, l'évaluation du projet et la mise en œuvre éventuelle de mesures compensatoires.

Dans un autre champ, plusieurs auteurs ont montré le rôle et l'importance de la culture, au sens large, dans la perception de l'environnement en tant que paysage. C'est notamment le philosophe Alain Roger (1997) qui a développé le principe d'«artialisation» du pays en paysage par le biais de représentations artistiques révélant les qualités esthétiques d'un site. Ou encore le géographe Augustin Berque (1996), qui a défini six critères caractérisant les sociétés paysagères. Ces critères sont les suivants :

«1. Une littérature (orale ou écrite) chantant la beauté des lieux [...];

2. des jardins d'agrément ;

3. une architecture aménagée pour jouir d'une belle vue ;

4. des peintures représentant l'environnement;

5. un ou des mots pour dire « paysage»;

6. une réflexion explicite sur le " paysage.»

Nous nous appuierons, entre autres, sur ces critères pour retenir trois objectifs de qualité paysagère dans la pratique opérationnelle de l'urbanisme: la prise en compte de la qualité paysagère dans les documents de cadrage des projets urbains (depuis la phase de conception jusqu'à la réalisation), la présence de jardins ou d'espaces publics de détente au sein du projet urbain, l'intégration des projets de construction ou de rénovation dans le paysage de la rue.

Le référentiel «Un aménagement durable pour Paris », développé par la ville de Paris (2007), présente 21 objectifs de développement durable applicables à l'échelle du projet urbain et propose une méthode d'évaluation en phases de conception, de travaux et de gestion, basée sur une série d'indicateurs correspondant à chacun de ces objectifs. La 
prise en compte de la qualité paysagère a été tardivement intégrée dans l'axe 2 du référentiel portant sur «Un cadre urbain vivant et chaleureux» et plus particulièrement dans le chapitre 6 "Améliorer la qualité paysagère de l'espace urbain ». Il nous est en effet apparu que les démarches de développement durable, y compris celle proposée par le référentiel de la ville de Paris, ne prennent que rarement en considération les effets des actions recommandées sur le paysage.

11 C'est la raison pour laquelle nous avons cherché à intégrer cette dimension paysagère dans la conception des projets urbains en nous inspirant des principes adoptés par la Convention européenne du paysage et l'approche «culturaliste». Nous partons de l'hypothèse suivant laquelle il serait envisageable d'introduire la notion de qualité paysagère dans les modes actuels de conception urbaine, comme un véritable objectif de projet urbain, au même titre que les objectifs de développement durable.

12 La volonté de renforcer une telle approche provient notamment des difficultés rencontrées par les acteurs de l'aménagement parisiens à faire cohabiter le patrimoine et le renouvellement urbain durable.

13 Ainsi, à Paris, une véritable acception de la notion de qualité paysagère au cours du suivi des projets urbains nous semble pouvoir accompagner la nécessaire cohérence entre contraintes patrimoniales et contraintes environnementales.

14 La démarche proposée, au sein du référentiel, et au croisement des deux approches précitées, s'appuie sur un choix de huit indicateurs, dont un dernier portant sur la compensation, qui n'est à utiliser qu'en cas de non-atteinte évidente des objectifs de qualité paysagère. Ces indicateurs ont été définis pour déterminer la capacité d'une opération d'aménagement à « faire » paysage urbain.

15 À l'instar des indicateurs d'évaluation du référentiel parisien, nous proposons de doter chacun de ces sept (+ un) critères d'une échelle de notation variant de 1 (note la plus basse) à 5 (note la plus élevée) car elle permet d'uniformiser les évaluations en limitant le risque de subjectivité.

16 Les indicateurs de qualité paysagère retenus sont les suivants :

17 Critère $\mathrm{n}^{\circ} 1$ Échelle de notation

\begin{tabular}{|l|l|}
\hline $\begin{array}{l}\text { Un diagnostic sur la qualité } \\
\text { paysagère du site }\end{array}$ & $\begin{array}{l}1 \text { ou 2. Aucun diagnostic sur la qualité paysagère du site ou une } \\
\text { approche très sommaire. } \\
\text { 3. Un diagnostic sur la qualité paysagère du site. } \\
4 \text { ou } 5 . \text { Un diagnostic sur la qualité paysagère du site complété par } \\
\text { une étude des motifs à valoriser ou à renforcer. }\end{array}$ \\
\hline
\end{tabular}

Critère $\mathrm{n}^{\circ} 2$ Échelle de notation 
La présence de jardins ou d'espaces publics au sein du projet urbain
1 ou 2. Aucun espace de promenade/détente à moins de 300 mètres de tout logement inscrit dans le périmètre du projet.

3. Un espace de promenade/détente à moins de 300 mètres de tout logement inscrit dans le périmètre du projet.

4 ou 5 . Au moins un espace de promenade à moins de 300 mètres de tout logement inscrit dans le périmètre du projet avec accessibilité sur voie piétonne et/ou réservée aux circulations douces (type zone partagée ou zone 30).

Critère $n^{\circ} 3$ Échelle de notation

\begin{tabular}{|l|l|}
\hline L'intégration des mobiliers urbains, de la publicité \\
$\begin{array}{l}\text { et des enseignes lumineuses dans le paysage de la } \\
\text { rue }\end{array}$ & $\begin{array}{l}1 \text { ou 2. Des aménagements d'espaces publics } \\
\text { mal ou peu intégrés dans le paysage de la rue. } \\
3 . \text { Des aménagements d'espaces publics } \\
\text { préservant le paysage de la rue. } \\
4 \text { ou 5. Des aménagements d'espaces publics } \\
\text { préservant et/ou valorisant le paysage de la } \\
\text { rue. }\end{array}$ \\
\hline
\end{tabular}

\begin{tabular}{|l|l|}
\hline $\begin{array}{l}\text { L'intégration des projets de construction ou de } \\
\text { rénovation dans le paysage de la rue }\end{array}$ & $\begin{array}{l}1 \text { ou 2. Une ou des architectures mal ou peu } \\
\text { intégré(s) dans le paysage de la rue. } \\
\text { 3. Une ou des architectures préservant le } \\
\text { paysage de la rue. } \\
4 \text { ou 5. Une ou des architectures préservant et/ } \\
\text { ou valorisant le paysage de la rue. }\end{array}$ \\
\hline
\end{tabular}

\begin{tabular}{|l|l|}
\hline & $\begin{array}{l}1 \text { ou 2. Aucune ou des représentations trop partielles montrant } \\
\text { l'intégration du projet urbain dans le site. } \\
\text { L'intégration du projet } \\
\text { urbain dans le site et les représentations montrant l'intégration du projet urbain dans le } \\
\text { territoire élargi }\end{array}$ \\
$\begin{array}{l}\text { site à l'état initial et à l'état projeté. } \\
\text { 4 ou 5. Des représentations montrant l'intégration du projet urbain } \\
\text { dans le site à l'état initial et à l'état projeté complétées par une } \\
\text { approche territoriale (échelle du quartier ou de la ville), voire } \\
\text { métropolitaine. }\end{array}$ \\
\hline
\end{tabular}

\section{Critère $n^{\circ} 6$ Échelle de notation}




\begin{tabular}{|c|c|}
\hline $\begin{array}{l}\text { La prise en compte des caractéristiques } \\
\text { paysagères citées dans les documents politiques } \\
\text { et réglementaires du territoire de projet }\end{array}$ & $\begin{array}{l}1 \text { ou 2. Aucune analyse des caractéristiques } \\
\text { paysagères à préserver dans les documents } \\
\text { politiques ou réglementaires. } \\
\text { 3. Une prise en compte explicite des } \\
\text { caractéristiques paysagères à préserver dans les } \\
\text { documents politiques ou réglementaires. } \\
4 \text { ou } 5 \text {. Une prise en compte supérieure aux } \\
\text { caractéristiques paysagères à préserver dans les } \\
\text { documents politiques ou réglementaires. }\end{array}$ \\
\hline
\end{tabular}

Critère $\mathrm{n}^{\circ} 7$ Échelle de notation

\begin{tabular}{|c|c|}
\hline $\begin{array}{l}\text { La prise en compte de la qualité } \\
\text { paysagère dans les documents de } \\
\text { cadrage de l'opération }\end{array}$ & $\begin{array}{l}1 \text { ou 2. Aucune prise en compte de la qualité paysagère } \\
\text { dans les documents de cadrage de l'opération. } \\
\text { 3. Une prise en compte explicite de la qualité paysagère } \\
\text { dans les documents de cadrage de l'opération. } \\
4 \text { ou } 5 \text {. Une prise en compte de la qualité paysagère } \\
\text { supérieure aux objectifs définis dans les documents de } \\
\text { cadrage de l'opération. }\end{array}$ \\
\hline
\end{tabular}

\section{Critère $\mathrm{n}^{\circ} 8$ Échelle de notation}

\begin{tabular}{|c|c|}
\hline $\begin{array}{l}\text { En cas de non-atteinte des objectifs } \\
\text { de qualité paysagère } \\
\text { visés, la mise en œuvre de mesures } \\
\text { compensatoires en faveur de la qualité } \\
\text { paysagère }\end{array}$ & $\begin{array}{l}\text { 1. Aucune mesure compensatoire mise en place. } \\
\text { 2. La mise en place de mesures compensatoires sur un } \\
\text { autre territoire situé dans une autre collectivité. } \\
\text { 3. La mise en place de mesures compensatoires sur un } \\
\text { autre territoire situé dans la collectivité ou le } \\
\text { quartier. } \\
4 \text { ou 5. La mise en place de mesures compensatoires } \\
\text { dans le périmètre du projet urbain. }\end{array}$ \\
\hline
\end{tabular}

L'approche paysagère étant globale et complexe par définition, elle ne peut se limiter à sept (+ un) indicateurs si pertinents soient-ils. Aussi, un croisement de l'analyse paysagère avec des démarches patrimoniales, environnementales, de participation citoyenne, d'innovation urbaine, nous apparaît essentiel pour couvrir les champs relatifs à la qualité paysagère en particulier et au paysage urbain en général.

C'est la raison pour laquelle l'évaluation de la qualité paysagère d'une opération d'aménagement présente la difficulté de devoir croiser les résultats obtenus sur les sept indicateurs de qualité paysagère avec ceux des autres thématiques, à la fois sensibles, qualitatives et quantitatives, qui contribuent également à la qualité du cadre de vie. Ces « autres » thématiques, citées dans le référentiel parisien, sont les suivantes : 
Figure 1. Objectifs de développement durable contribuant à la qualité du cadre de vie

\begin{tabular}{|c|c|}
\hline Indicateurs & $\begin{array}{l}\text { Prise en compte dans le } \\
\text { référentiel }\end{array}$ \\
\hline 1. La concertation et les échanges avec les habitants. & Objectif 3 (axe 1 ) \\
\hline 2. L'innovation et l'expérimentation de nouvelles formes urbaines & Objectif 4 (axe 1) \\
\hline 3. La préservation du patrimoine bâti et naturel & Objectifs 9 et 10 (axe 2 ) \\
\hline 4. Le choix de matériaux écologiques & Objectif 18 (axe 3 ) \\
\hline $\begin{array}{l}\text { 5. L'intégration des contraintes liées à la gestion des programmes dans } \\
\text { le temps }\end{array}$ & Objectif 5 (axe 1) \\
\hline $\begin{array}{l}\text { 6. La capacité des espaces publics à créer des liens sociaux et un cadre } \\
\text { de vie de qualité }\end{array}$ & Objectifs 6 et 7 (axe 2) \\
\hline $\begin{array}{l}\text { 7. L'amélioration de la biodiversité, quantité et qualité des espèces } \\
\text { végétales présentes sur le site }\end{array}$ & Objectif 12 (axe 3 ) \\
\hline 8. La gestion de l'eau & Objectif 15 (axe 3) \\
\hline
\end{tabular}

Source : extrait du référentiel « Un aménagement durable pour Paris »/ville de Paris DU৫.

La dimension "sensible» du paysage, évoquée aux points un et six, pourrait être approfondie en s'appuyant sur une analyse détaillée de la perception paysagère des usagers de l'espace public et utilisateurs des bâtiments. Des enquêtes sociologiques et anthropologiques appropriées auraient ainsi pour fonction de préciser les catégories d'usagers (habitants, commerçants, étudiants, actifs, retraités, touristes, etc.) présents sur le site, et les différents profils d'acteurs (maîtrise d'ouvrage publique et privée, maîtrise d'œuvre, associations locales, conseil de quartier, etc.) participant à l'élaboration d'un projet urbain.

Néanmoins, dans le cadre de cet article, nous proposons de nous en tenir aux sept (+ un) indicateurs définis plus haut et d'en vérifier les modalités de mise en œuvre opérationnelle en les appliquant sur trois opérations d'aménagement parisiennes.

\section{Analyse comparative de la qualité paysagère dans trois projets urbains}

Les opérations étudiées ont été retenues du fait de leur évolution dans des calendriers parallèles et de leurs dimensions relativement similaires, mais présentant des contextes urbains et des programmations différents. Il s'agit de :

- la zone d'aménagement concertée (ZAC) de la gare de Rungis (13e arrondissement), qui s'inscrit dans une réflexion à dominante d'urbanisme fonctionnel et participatif portant sur une approche environnementale et sociale ;

- la ZAC Pajol (18 arrondissement), qui intègre explicitement la question paysagère et la décline sur des associations entre patrimoine bâti, espace public et jardin ; 
- du secteur Fréquel-Fontarabie ( $20^{\mathrm{e}}$ arrondissement), marqué par une volonté politique locale et des ambitions énergétiques fortes, qui recherche la qualité du cadre de vie sans pour autant faire appel à la notion de paysage. cour du site (figure 2), qui contribuera à améliorer la qualité paysagère du quartier et le cadre de vie des usagers. De plus, suite à l'étude sur la mobilité, la circulation a été fortement réduite par rapport aux objectifs initiaux du projet réduisant ainsi les impacts des nuisances (pollution atmosphérique, bruit...) dans le quartier. Les aménagements des espaces publics intègrent une dimension environnementale et sociale en proposant une station Vélib', une place et une voie piétonnes ainsi qu'une aire de jeux de ballons et de sports de glisse (roller, skate, etc.).

Figure 2. Plan du jardin

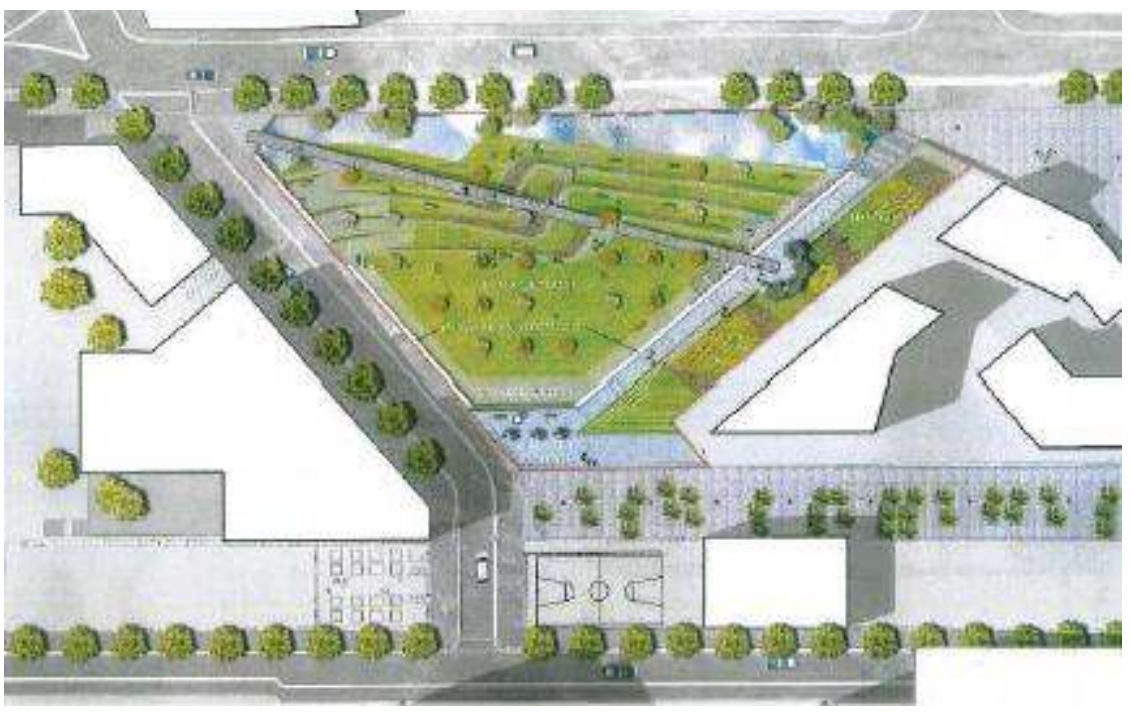

Source : Michel Péna, paysagiste.

La programmation de la ZAC de Rungis comprend différents bâtiments (logements étudiants, établissement hébergeant des personnes âgées dépendantes, crèche, haltegarderie, bureaux) aux architectures variées, qui donneront une nouvelle identité à ce quartier.

En cours de conception, ceux-ci ont fait l'objet de nombreuses représentations (plansmasses, élévations, photomontages, perspectives,...), qui ont évolué au fur et à mesure de l'avancement des projets et ont montré ce que les bâtiments et le site dans son ensemble pourraient «donner à voir» une fois achevés. À titre d'exemple, la 
perspective du bâtiment de bureaux, ci-dessous, représente la rue Brillat-Savarin après les travaux.

Figure. 3. Immeuble de bureaux

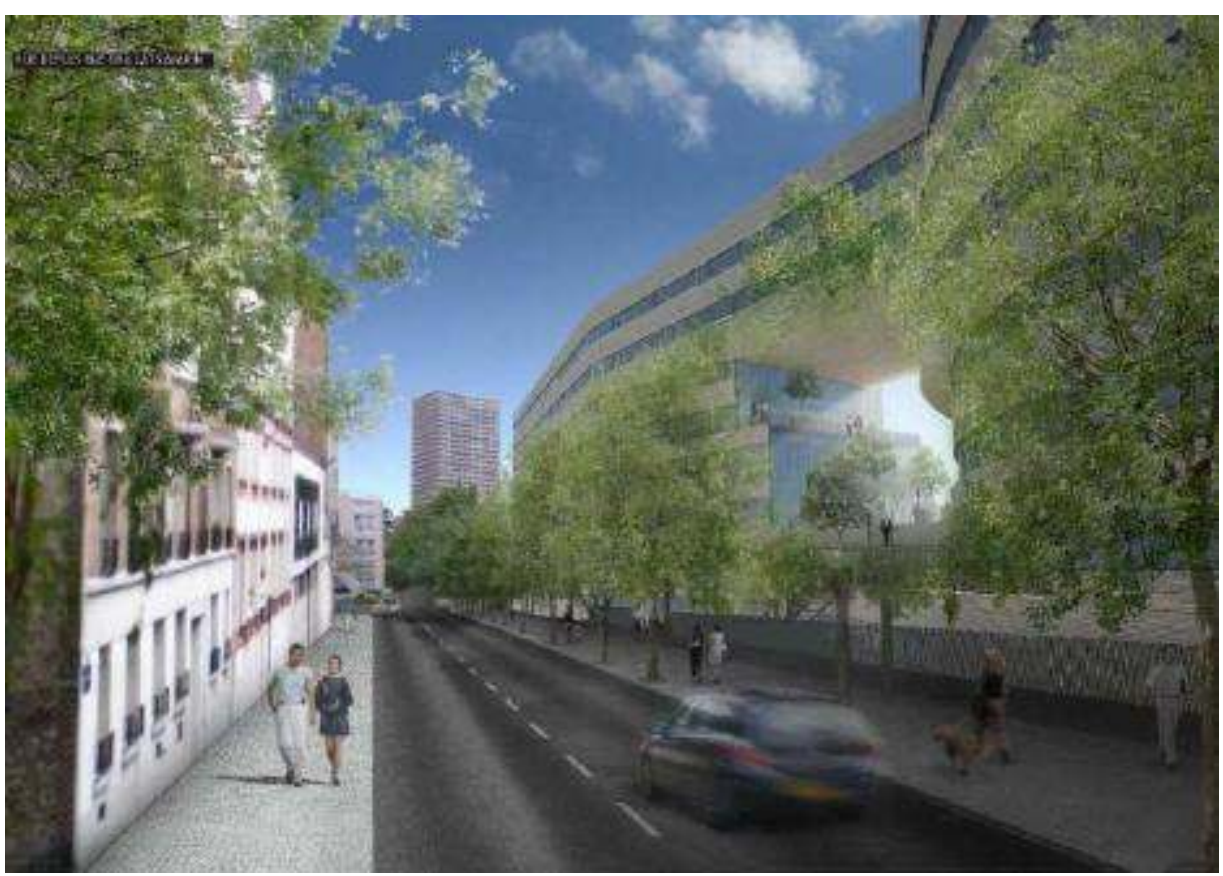

Source : ICADE, maître d'ouvrage et MVRDV Winy MAAS, architecte

Au cours du suivi de cette opération, la notion de paysage est apparue dès la phase de conception. Ainsi, dans une note rédigée par l'aménageur, l'objectif portant sur la " préservation et la valorisation du paysage sur le site » est évoqué dans un paragraphe portant sur l' « amélioration de la qualité de l'environnement local ».

En revanche, il n'existe pas d'étude témoignant d'une réflexion sur l'inscription paysagère du projet à l'échelle du quartier, de l'arrondissement ou même à l'échelle métropolitaine. Malgré cela, la tardive mais réelle prise en compte d'une démarche de développement durable dans le suivi de cette opération, parmi les premières à Paris, lui confère une qualité paysagère indéniable.

Le graphique des sept critères d'évaluation de la qualité paysagère de la ZAC de Rungis se présente comme suit. 
Figure. 4. Qualité paysagère de la ZAC de la gare de Rungis ( $13^{\mathrm{e}}$ arrondissement)

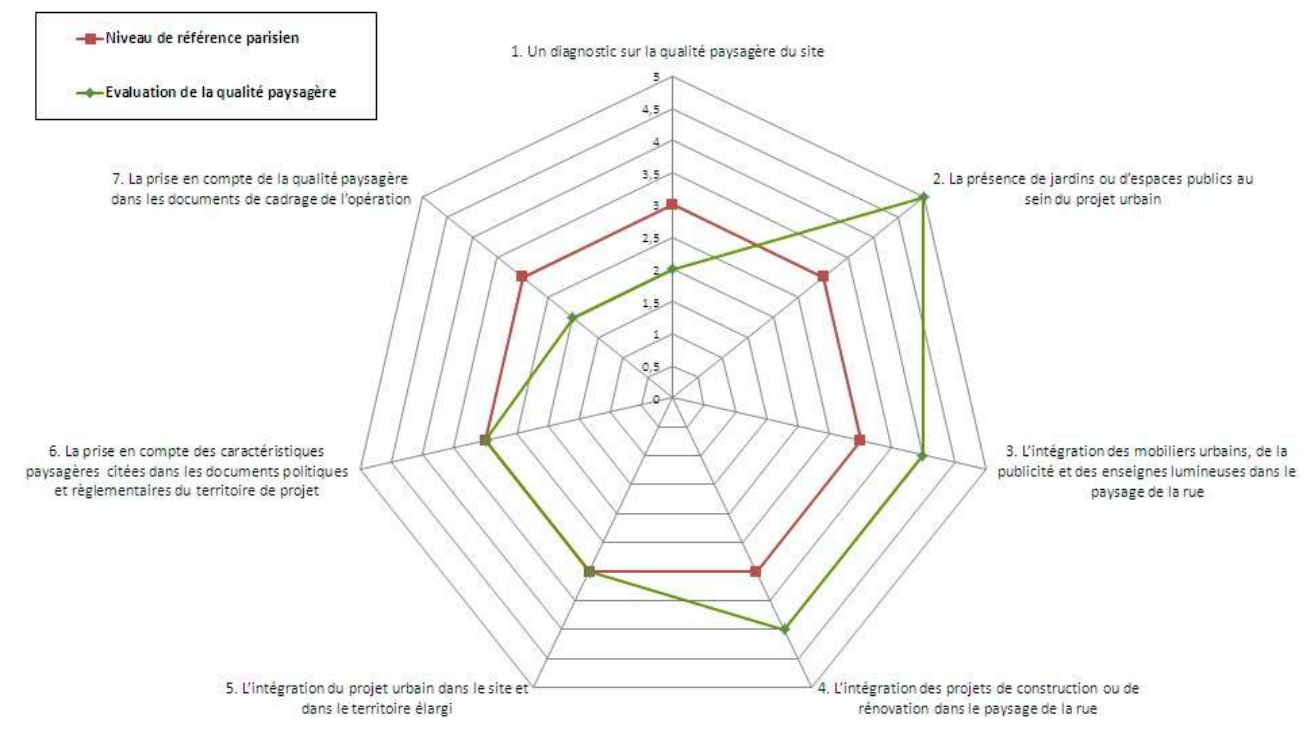

Le projet de la ZAC Pajol $\left(18^{\mathrm{e}}\right)$ a débuté en 2001 et est actuellement en phase de réalisation. Elle couvre une superficie de 3,3 hectares. La formation d'une association locale (Coordination Espace Pajol - CEPA), dès le début des études, ainsi que la rapide désignation d'un aménageur, tous deux très engagés en faveur d'une démarche de développement durable, ont favorisé la réalisation de nombreuses études techniques portant notamment sur :

- la préservation des caractéristiques paysagères et patrimoniales du site, liées à la présence de la grande halle identifiée comme un témoin du passé industriel ;

- l'alimentation énergétique et le développement des énergies renouvelables ;

- la récupération des matériaux de démolition et d'une part importante des structures préexistantes.

Deux documents de cadrage ont été rédigés pour préciser les objectifs de programmation et de développement durable de l'opération: la charte de développement durable et le cahier des prescriptions environnementales et de développement durable (CPEDD). Ces documents précisent les spécificités paysagères qu'il est recommandé de préserver et de valoriser dans le cadre du projet.

L'association CEPA s'est fortement investie auprès de la maitrise d'ouvrage pour contribuer à l'élaboration de la programmation et au suivi du projet. Ainsi, contrairement aux choix initialement retenus à la fin des années 1990, consistant en la création de logements avec démolition de la grande halle, il a été décidé de préserver et de réhabiliter ce patrimoine bâti. Cette réorientation du projet est considérée aujourd'hui comme un maillon essentiel de la réussite de l'opération.

Le programme comprend la réalisation d'équipements publics (bibliothèque, gymnase, collège), d'une auberge de jeunesse, d'un immeuble de bureaux, ainsi que la création d'un jardin semi-couvert, situé en partie sous la halle. 
Figure 5. Vue du jardin intérieur de la ZAC Pajol

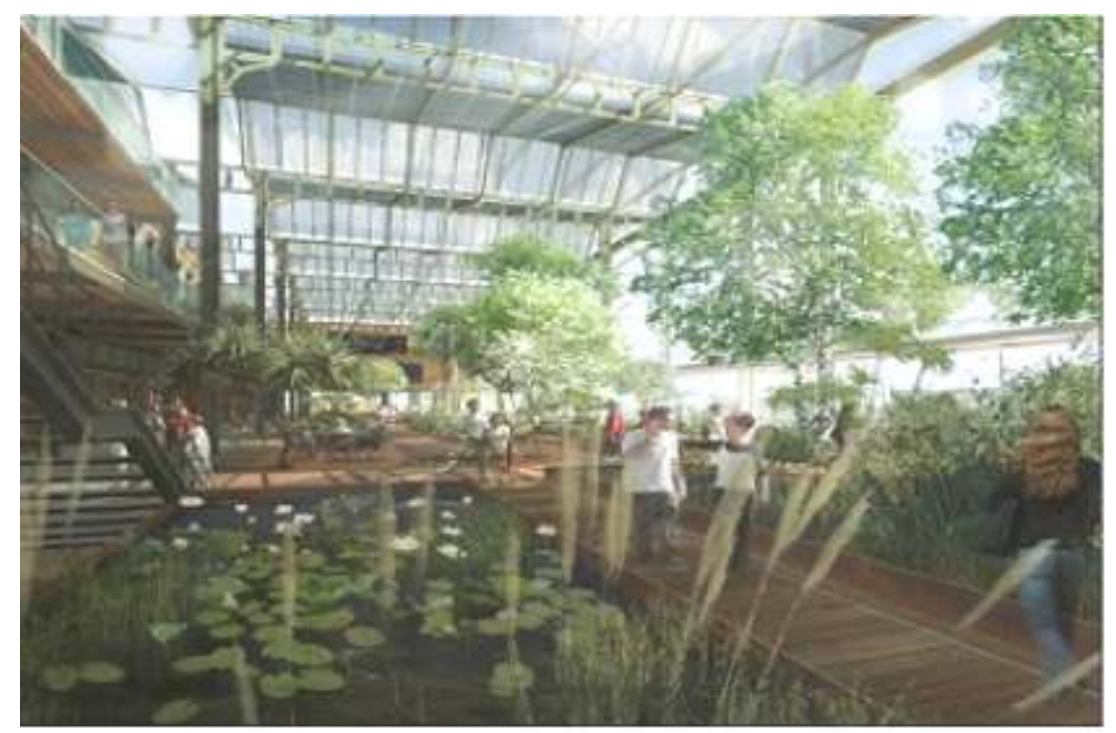

Source : Jourda architectes, Paris.

42 En complément, une vaste esplanade piétonne contribuera à améliorer la qualité de vie des habitants du quartier, tout en réduisant l'impact des nuisances. Ces aménagements répondent pleinement aux attentes des riverains et des associations locales, qui souhaitaient que cette opération pallie au déficit d'espaces publics dans le quartier. En outre, il est à souligner l'initiative originale de l'aménageur, qui a édité une revue mensuelle 22bis rue Pajol et a mis en place une frise le long de la rue Pajol pour présenter les différentes phases de transformation du site.

Figure 6. Affichage en frise sur la rue

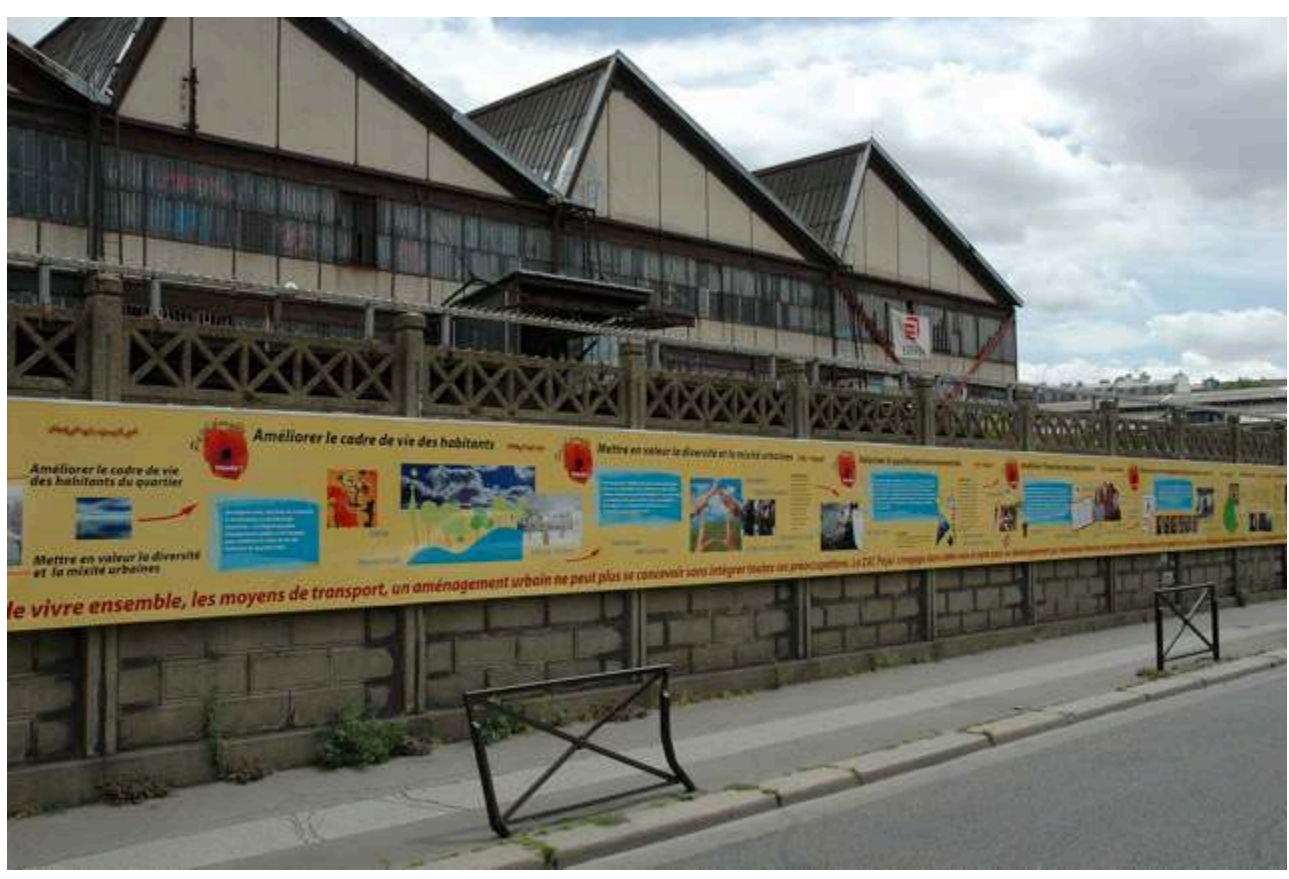

Source : Jacques Leroy, photographe, ville de Paris SCC/DU. 
À l'achèvement de l'opération, les vues du site, qui s'offriront aux usagers ou aux promeneurs, depuis les derniers étages des bâtiments, les ponts des rues Riquet ou du Département, présenteront divers motifs de paysage répondant ainsi à la notion de paysage urbain.

\section{Figure 7. Halle Pajol}

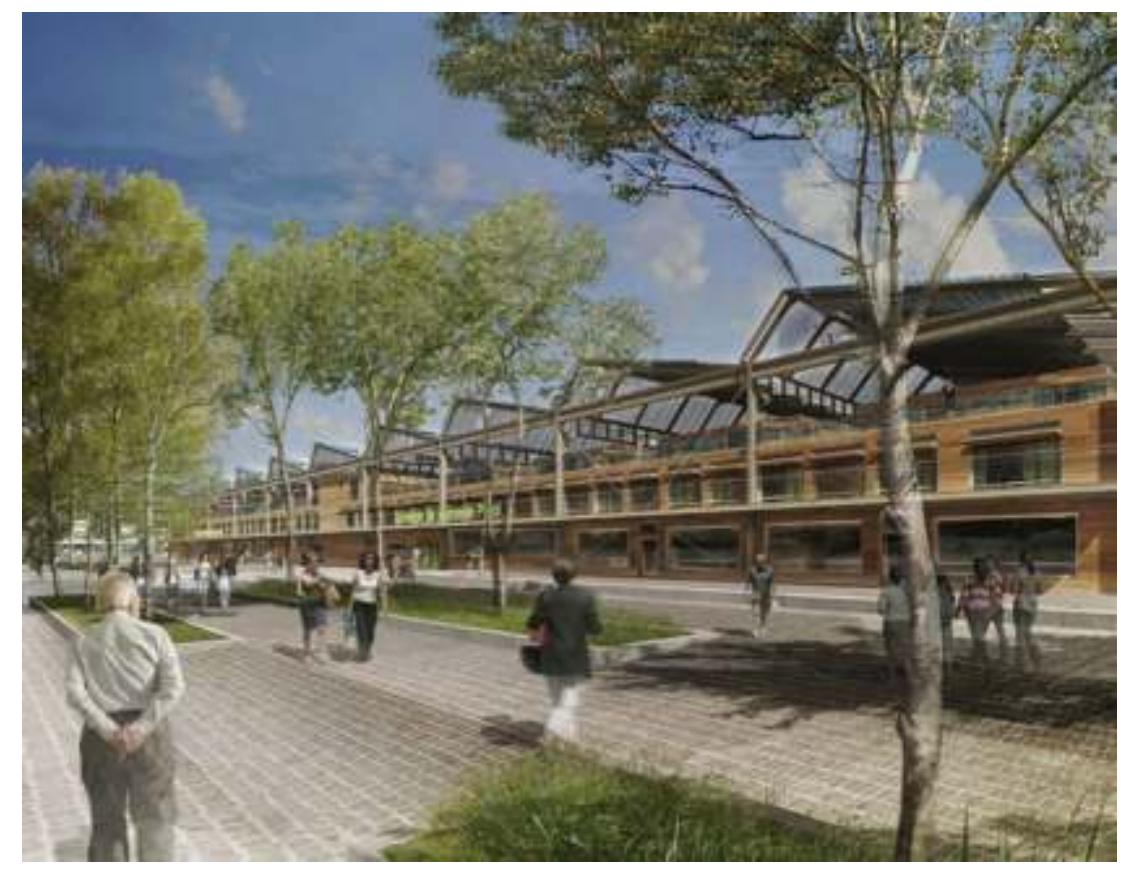

Source : Jourda architectes, Paris.

Le graphique des six critères d'évaluation de la qualité paysagère de la ZAC Pajol se présente comme suit.

Figure 8. Qualité paysagère de la ZAC Pajol (18e)

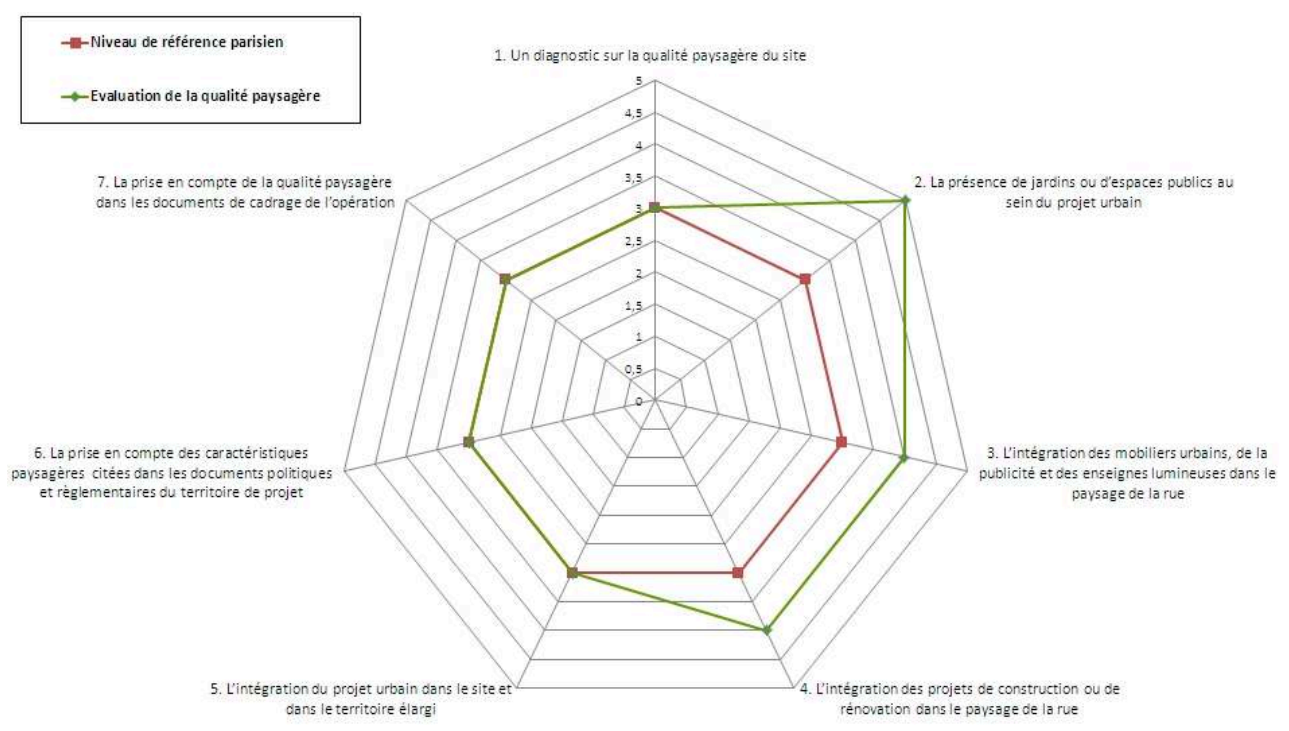

Le projet du secteur Fréquel-Fontarabie $\left(20^{\mathrm{e}}\right)$ a débuté en 2002 et est actuellement en phase de réalisation. Il couvre une superficie d'un hectare. Ce site a fait l'objet de 
nombreuses études portant notamment sur la résorption de l'insalubrité, qui était le motif initial du lancement de cette opération. De plus, les élus d'arrondissement ont exprimé, dès le départ, leur volonté de faire de ce projet une opération exemplaire en matière d'économie d'énergie. Ainsi, des études techniques portant sur l'orientation, l'ensoleillement et les enveloppes des bâtiments ont accompagné la conception urbaine du site. La maîtrise d'ouvrage a rédigé une charte de développement durable prenant en compte, de manière indirecte, la qualité paysagère dans son premier objectif: « Maîtriser les impacts du projet sur l'environnement extérieur ».

Par ailleurs, le projet a retenu le principe de préserver les espaces libres existant en cœur d'îlot pour réaliser un jardin public accessible par des passages sous-porche (figure 9). Ce nouvel espace, entièrement piéton, contribuera à améliorer le cadre de vie des usagers du quartier en leur offrant de nouveaux lieux de promenade, de détente et de repos.

Figure 9. Vue du jardin du secteur Fréquel-Fontarabie

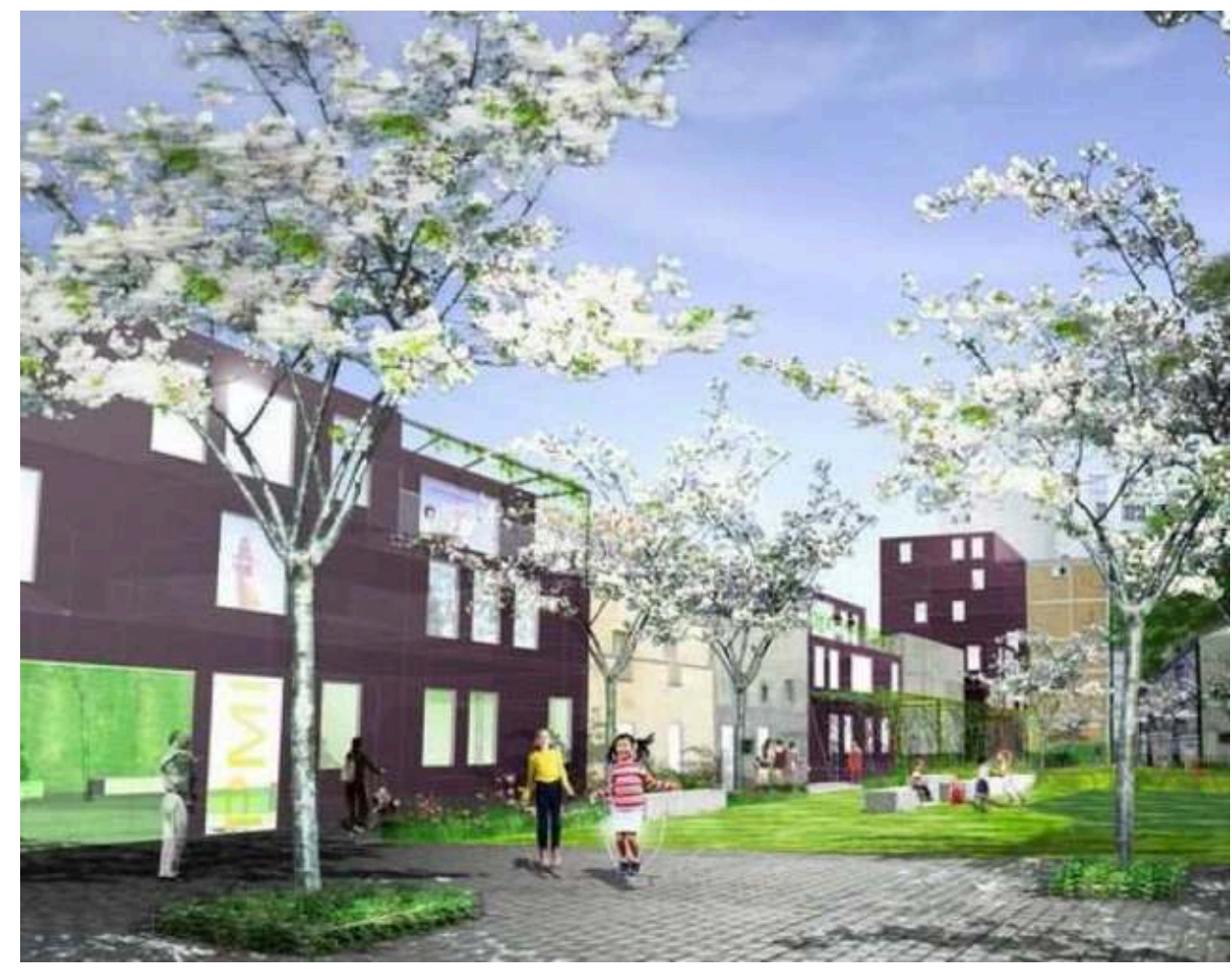

Source : Ville de Paris DEVE.

Le secteur Fréquel-Fontarabie est, en somme, une juxtaposition d'édifices, rénovés ou en cours de construction, mêlant des typologies faubouriennes et contemporaines, dans un contexte urbain "intimiste » car situé en cœur d'îlot. Ces différents bâtiments apportent un nouveau regard sur ce site en devenir. 
Figure 10. Secteur Fréquel Fontarabie

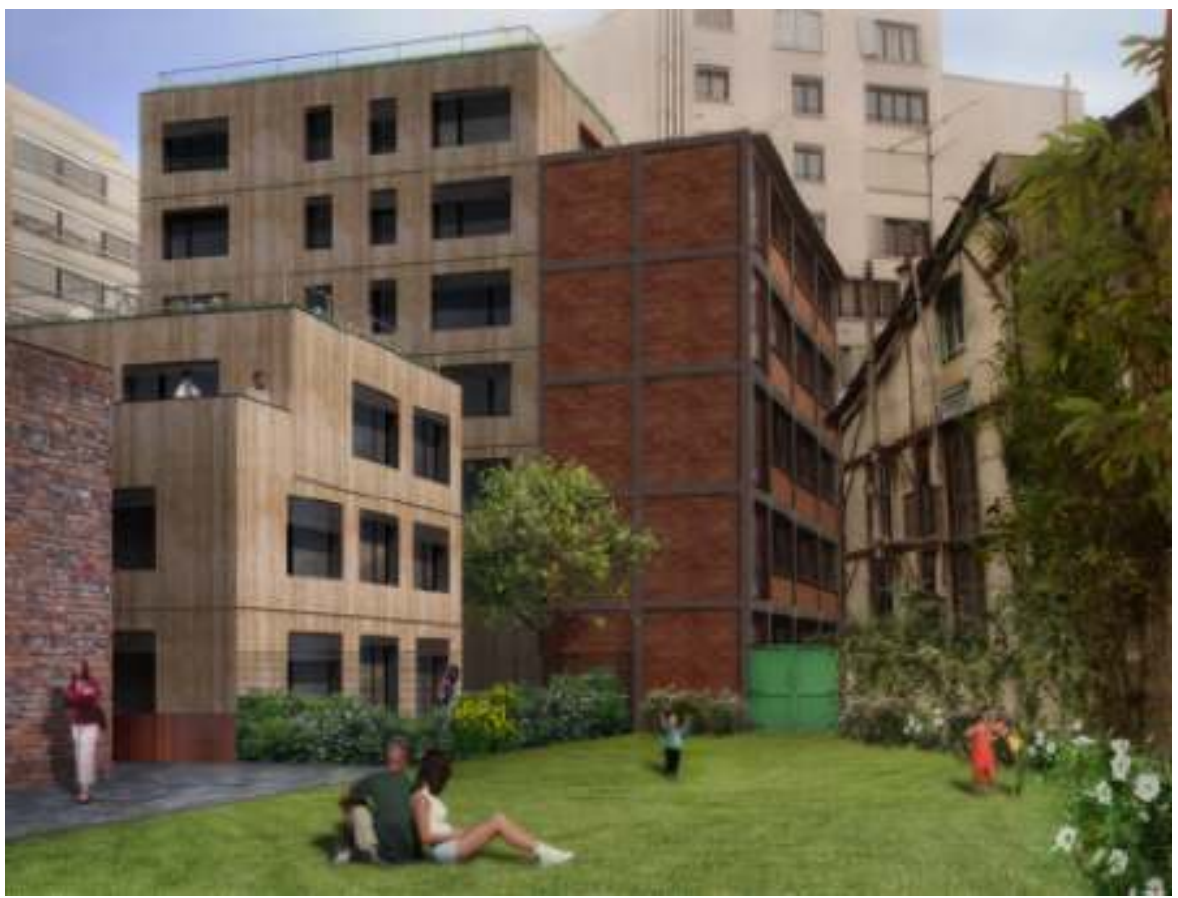

Source : Atelier Pascal Gontier, architectes.

Le graphique des six critères d'évaluation de la qualité paysagère du secteur FréquelFontarabie se présente comme suit.

Figure 11. Qualité paysagère du secteur Fréquel-Fontarabie (20e)

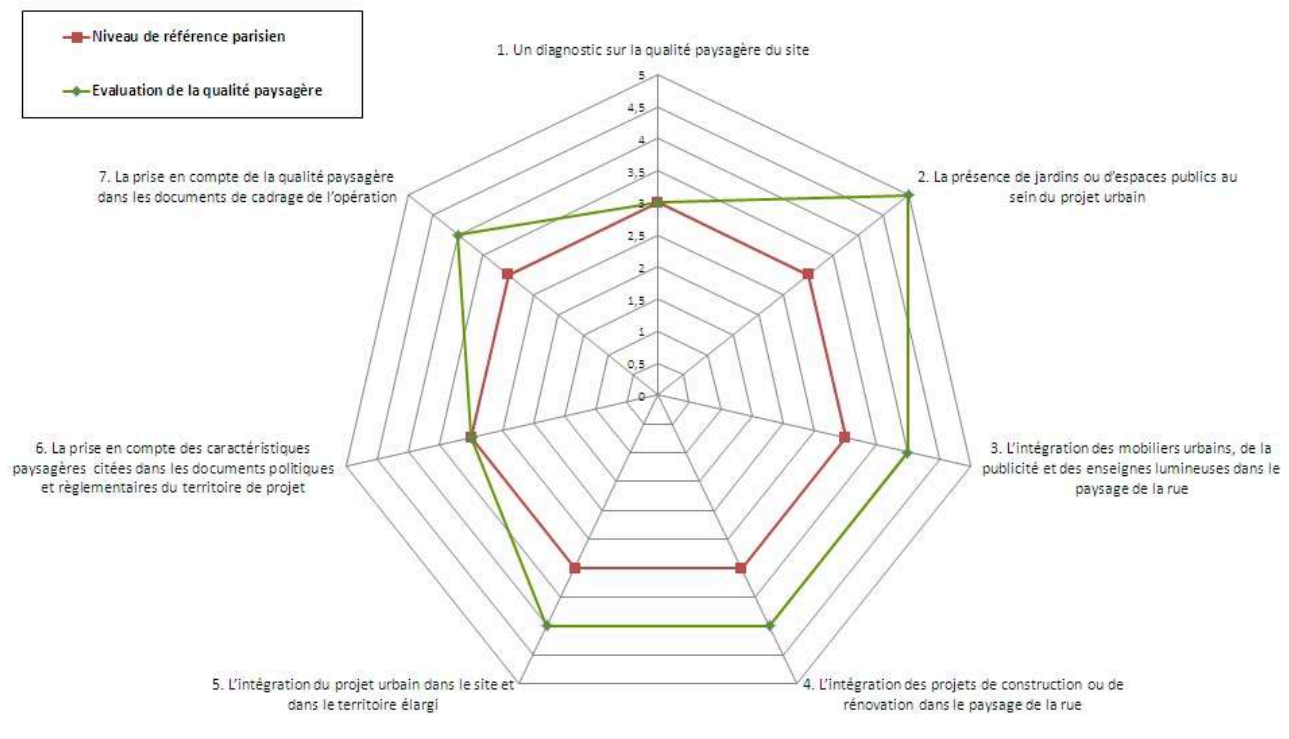

\section{De la qualité paysagère des opérations d'aménagement à l'approche globale}

Ces premières analyses nous ont permis de formaliser, sous la forme de critères d'évaluation, les étapes nécessaires à la mise en œuvre d'un certain niveau de qualité 
paysagère dans les projets urbains durables. La prise en compte de ces différents critères, à un instant $t$ de l'avancement du projet, apparaît comme une piste de réflexion pour accompagner la constitution d'un paysage urbain durable (PUD).

Ainsi, les sept (+ un) indicateurs développés ci-dessus, lorsqu'ils sont inscrits dans la méthode d'évaluation du référentiel développé par la ville de Paris, permettent de qualifier la thématique " qualité paysagère du quartier » ou du moins d'en donner une certaine réalité concrète.

De même, une extraction similaire avec les indicateurs portant sur la concertation, l'énergie ou encore la biodiversité peut permettre d'analyser le niveau de performance d'une opération d'aménagement dans des thématiques spécifiques. Ces évaluations peuvent alors apparaitre en complément des évaluations globales des opérations pour présenter, de manière plus fine, les résultats obtenus dans des champs spécifiques.

Pour exemple, le diagramme de l'évaluation globale de la ZAC Pajol (sur les 21 objectifs) synthétise la performance de la qualité paysagère dans l'objectif « Améliorer la qualité paysagère de l'espace urbain » (figure 12) et complète ainsi la compréhension globale des résultats de cette opération.

Figure 12. Radar d'évaluation de la ZAC Pajol en phase conception-réalisation

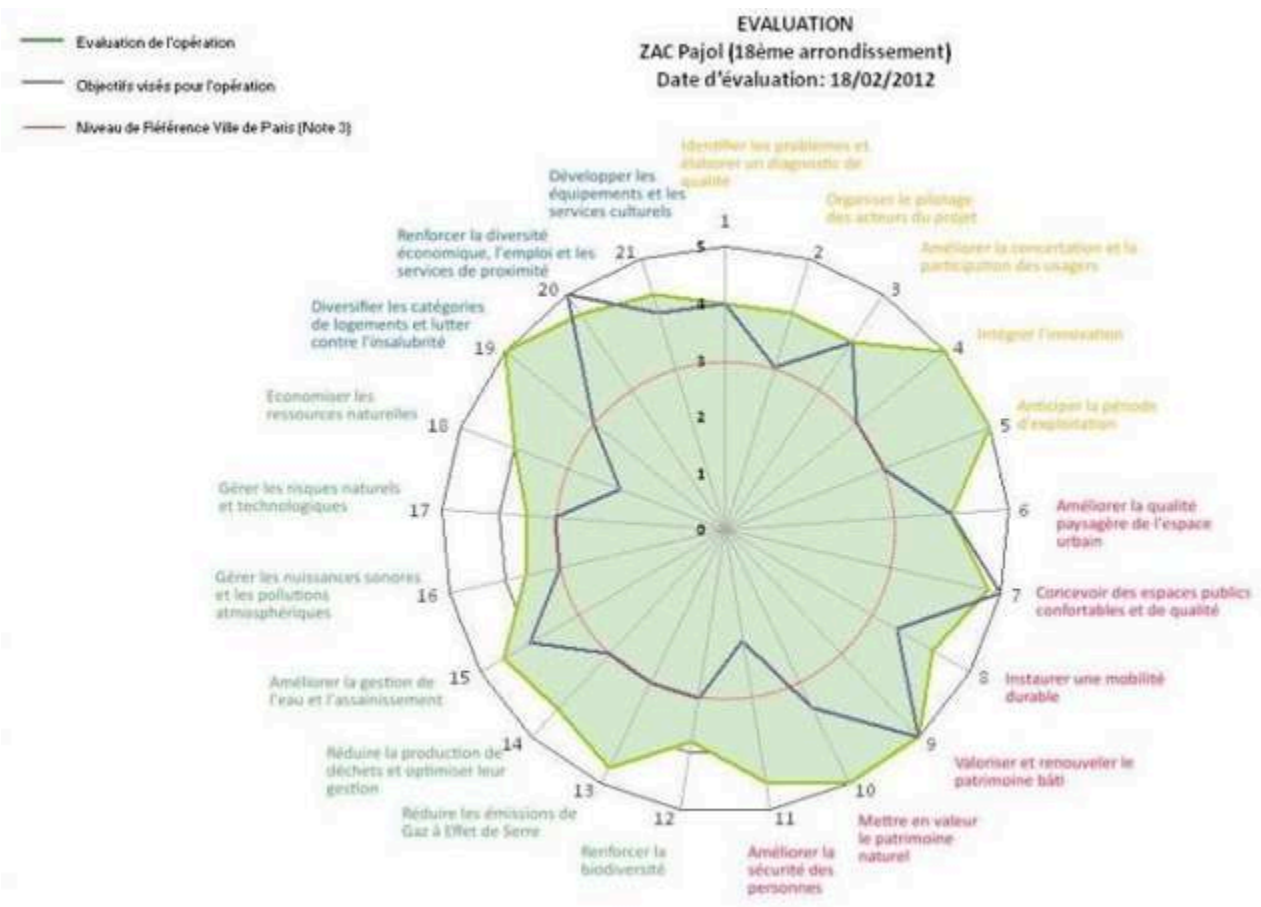

Source : Ville de Paris DU.

Le croisement entre les différentes thématiques et la «contextualisation » des évaluations, suivant les contraintes urbaines, politiques, sociologiques, ou géographiques propres à l'opération d'aménagement, permettent de montrer plusieurs "facettes» des opérations d'aménagement, notamment lorsque ces différentes évaluations sont réalisées régulièrement au fur et à mesure de l'avancement des projets. 


\section{Conclusion} qualité paysagère apparaisse comme un véritable objectif de projet urbain durable ? Cet objectif pourrait-il être évalué dès la phase de conception des projets urbains ou des projets de construction, dans le cadre des jurys de concours par exemple? Quel pourrait être le suivi d'un tel objectif en phase de réalisation puis de gestion du quartier?

La qualité paysagère contribuant à la construction de paysages urbains durables, cette dernière est apparue, à travers notre recherche, comme une construction mentale intégrée à la notion de ville durable visant à pallier les effets perçus comme nocifs du développement urbain. La ville durable serait alors porteuse d'espoirs d'une ville réhabilitée et construite pour mieux s'adapter au changement climatique, en continuité et en harmonie avec la ville existante, ainsi que dans le respect de la nature, de la cohésion sociale et économique des paysages urbains. 


\section{BIBLIOGRAPHIE}

Berque, A., « Le Principe de Zong Bing - paysage et dépassement de la modernité », Horizon paysage, 2001.

Berque, A., « Le sauvage construit », Ethnologie française, dossier « La nature en ville », 2009.

Berque, A., La Pensée paysagère, Paris, Édition Archibooks, coll. « Crossborders », Paris, 2008.

Berque, A., Conan, M., Donadieu, P., Lassus, B., Roger, A., Mouvance. Cinquante mots pour le paysage, Paris, Éditions de la Villette, 1999.

Berque, A., Les Raisons du paysage. De la Chine antique aux environnements de synthèse, Paris, Hazan, 1996.

Clergeau, P., Une écologie du paysage urbain, Rennes, Les Éditions Apogée, 2007.

Donadieu, P., Paysage, entre natures et cultures, Paris, édition Armand Colin, 2007.

Emelianoff, C. (coordonné par), « Urbanisme durable? ", Écologie \& Politique, Paris, Éditions Syllepse, 2004.

Rimbert, S., "Contribution à la méthodologie cartographique et à l'observation des paysages urbains », thèse dirigée par H. Isnard, université de Strasbourg, 1972.

Roger, A., Court Traité du paysage, Paris, Gallimard, 1997.

\section{Rapports institutionnels}

Convention européenne du paysage, appelée également la Convention de Florence, série des traités du Conseil de l'Europe ${ }^{\circ}$ 176, Florence, 2000, entrée en vigueur en 2004.

Mairie de Paris, le PLU de Paris, voté par le Conseil de Paris en juin 2006.

Mairie de Paris, Cahier des recommandations environnementales pour les acteurs de la construction et de l'aménagement, février 2008.

Mairie de Paris, le PSMV du Marais, juillet 2003.

Mairie de Paris, le PSMV du $7^{\mathrm{e}}$ arondissement, juillet 2003.

Mairie de Paris, le référentiel « Un aménagement durable pour Paris », 2007.

Mairie de Paris, « L'environnement à Paris, une politique des actes », Cahier de l'environnement, $\mathrm{n}^{\circ} 1$, juin 2005

Rapport Gro Harlem Brundtland, Notre avenir à tous, WCED, Londres, Oxford University Press, 1987.

\section{RÉSUMÉS}

Alors que les démarches de développement durable se généralisent dans les projets d'aménagement urbains au point de devenir la nouvelle «norme», les réflexions sur les modalités d'évaluation sont encore en construction. En s'appuyant sur la démarche de suivi et d'évaluation des projets urbains, développée par la ville de Paris, cet article questionne la possibilité d'évaluer la qualité paysagère d'un site en référence aux spécificités des sociétés dites «à paysage » dont relève la société parisienne. Les propositions d'indicateurs d'évaluation sont 
étayées par des expérimentations sur trois projets urbains parisiens, qui montrent la pertinence mais également les difficultés de mise en œuvre opérationnelle d'une telle démarche.

While the initiatives of sustainable development become widespread in the urban projects of urban planning in the point to become the new "standard", the reflections on the modalities of evaluation are still under construction. By leaning on the approach of follow-up and evaluation of the urban projects, developed by the city of Paris, this article questions the possibility of estimating the landscaped quality of a site in reference to the specificities of societies said " in landscape " from which raises the Parisian society. The proposals of indicators of evaluation are supported by experiments on three Parisian urban projects, which show the relevance but also the difficulties of operational implementation of such an approach.

\section{INDEX}

Mots-clés : urbanisme, projet urbain, développement durable, qualité paysagère, évaluation

Keywords : urbanism, urban projet, sustainable development, landscape quality, evaluation

\section{AUTEUR}

\section{CEDISSIA ABOUT-DE CHASTENET}

Architecte-voyer, docteur en urbanisme, référente développement durable pour la direction de l'Urbanisme, mairie de Paris.

cedissia.dechastenet[at]paris[dot]fr 